\title{
Variations des caractéristiques physiques et mécaniques du bois de Balanites aegyptiaca en fonction de trois provenances
}

\author{
Dao DougabKa ${ }^{1,2,3}$ \\ Jean GÉRARD ${ }^{1,2}$ \\ Tikri BıANZEUBE ${ }^{3}$ \\ Morgane DENDONCKER ${ }^{4}$ \\ Caroline VINCKE ${ }^{4}$ \\ Rémy MARCHAL ${ }^{5}$ \\ Daniel GUIBAL ${ }^{1,2}$ \\ Alban GuYot ${ }^{1,2}$ \\ ${ }^{1}$ Cirad \\ UPR BioWooEB \\ 34398 Montpellier \\ France \\ 2 BioWooEB \\ Univ Montpellier, Cirad \\ Montpellier \\ France
}

${ }^{3}$ Université polytechnique de Mongo

BP 4377

N'Djamena

Tchad

${ }^{4}$ Earth and Life Institute

Université catholique de Louvain

Croix du Sud, 2 L7.05.09

1348 Louvain-la-Neuve

Belgique

${ }^{5}$ HESAM Université

LABOMAP

Arts et Métiers Institute of Technology

71250 Cluny

France

Auteur correspondant /

Corresponding author:

Dao DOUGABKA -

dougabkadao@yahoo.fr

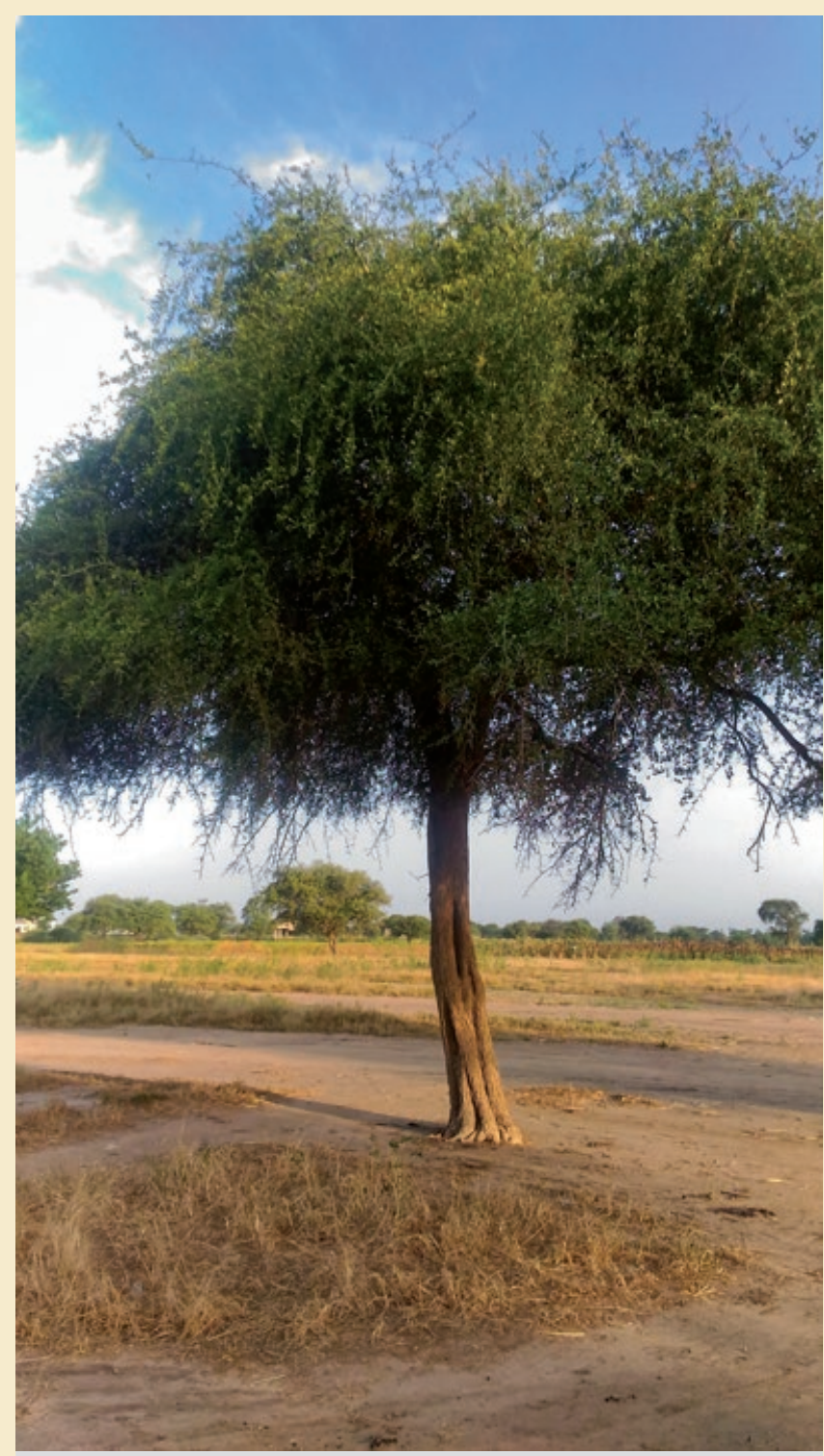

Photo 1.

Arbre $n^{\circ} 2$ (N’Djamena).

Tree No. 2 (N'Djamena).

Photo D. Dougabka.

Doi : 10.19182/bft2021.349.a36776 - Droit d'auteur (c) 2021, Bois et Forêts des Tropiques - (c) Cirad - Date de soumission : 15 janvier 2021 ; date d'acceptation : $1^{\text {er }}$ avril 2021 ; date de publication : $1^{\text {er }}$ septembre 2021.

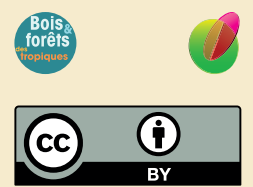

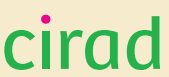

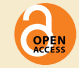

Licence Creative Commons :

Attribution - 4.0 International.

Attribution-4.0 International (CC BY 4.0)
Citer l'article / To cite the article

Dougabka D., Gérard J., Bianzeube T., Dendoncker M., Vincke C., Marchal R., Guibal D., Guyot A., 2021. Variations des caractéristiques physiques et mécaniques du bois de Balanites aegyptiaca en fonction de trois provenances. Bois et Forêts des Tropiques, 349 : 5-19. Doi : https://doi. org/10.19182/bft2021.349.a36776 


\section{RÉSUMÉ}

\section{Variations des caractéristiques physiques et mécaniques du bois de Balanites aegyptiaca en fonction de trois provenances}

Balanites aegyptiaca est une espèce caractéristique et emblématique des zones sèches d'Afrique et d'Asie. Elle revêt une grande importance socio-économique dans toute sa zone naturelle de répartition du fait de ses multiples usages. Toutefois, les propriétés technologiques de son bois sont mal connues, d'où des utilisations parfois inappropriées mais qui pourraient être élargies. Afin de mieux adapter ses applications à ses caractéristiques, nous avons déterminé les indicateurs de stabilité physique (masse volumique, infradensité, retrait radial total, retrait tangentiel total, retrait volumique total et point de saturation des fibres) et les indicateurs de comportement mécanique (module d'élasticité longitudinal, contrainte de rupture en flexion et compression) de ce bois. Quatrevingt-treize éprouvettes prélevées dans 13 arbres provenant des zones sahélienne et soudanienne tchadiennes, et de la zone sahélienne sénégalaise ont été testées. Les résultats obtenus montrent que, pour les trois provenances, le bois de $B$. aegyptiaca est mi-lourd $\left(797 \mathrm{~kg} / \mathrm{m}^{3}\right)$ avec une stabilité dimensionnelle moyenne : l'anisotropie de retrait est supérieure à $2(2,2)$, le retrait radial total et le retrait tangentiel total sont moyens, respectivement égaux à $4 \%$ et $8,5 \%$. Ses caractéristiques mécaniques sont moyennes (contraintes de rupture en compression et flexion statique respectivement égales à 49,4 MPa et 104,5 MPa) à faible (module d'élasticité longitudinal de $10473 \mathrm{MPa}$ ). Une comparaison des résultats obtenus en fonction des zones de prélèvement a mis en évidence des tendances variables selon les caractéristiques étudiées. Ces variations entre les trois provenances restent cependant limitées. Les résultats de l'étude montrent que le bois de $B$. aegyptiaca pourrait être utilisé de façon appropriée sous forme de matériau pour une plus large gamme d'emplois, sous réserve de la mise en place d'une gestion adaptée permettant sa restauration par plantation.

Mots-clés : Balanites aegyptiaca, bois, propriétés mécaniques, propriétés physiques, provenance sahélienne, provenance soudanienne, Sénégal, Tchad.

\section{ABSTRACT}

\author{
Variations in the physical and \\ mechanical properties of Balanites \\ aegyptiaca wood from three \\ provenances
}

Balanites aegyptiaca is a characteristic and emblematic tree species native to the dry zones of Africa and Asia. It has considerable socio-economic importance throughout its natural range of distribution because of its multiple uses. Despite this, the technological properties of its wood are not well known, hence sometimes inappropriate uses but potential for others. In order to match applications to characteristics of the wood, we determined physical stability indicators (density, infradensity, total radial shrinkage, total tangential shrinkage, total volumetric shrinkage and fibre saturation point) and mechanical behaviour indicators (longitudinal modulus of elasticity, bending strength and compression strength). Ninety-three specimens taken from 13 trees from the Sahelian and Sudanian zones in Chad and the Sahelian zone in Senegal were tested. Our results show that $B$. aegyptiaca wood from these three provenances is medium-heavy $(797 \mathrm{~kg} /$ $\mathrm{m}^{3}$ ) with moderate dimensional stability: shrinkage anisotropy is above 2 (2.2) and total radial and tangential shrinkage rates are medium at $4 \%$ and $8.5 \%$ respectively. Its mechanical characteristics are medium (static bending and compression strength at 49.4 MPa and 104.5 MPa respectively) to weak (longitudinal modulus of elasticity at $10,473 \mathrm{MPa}$ ). A comparison of the results obtained according to the provenance of the specimens showed variable tendencies depending on the characteristics analysed, although the variations between the three provenances are limited. Our study results show that the use of $B$. aegyptiaca wood as a material could be appropriate for a wider range of purposes, on condition that suitable management methods are in place with a view to their restoration by planting.

\section{Keywords: Balanites aegyptiaca,} wood, mechanical properties, physical properties, Sahelian provenance, Sudanian provenance, Senegal, Chad.

\author{
Variaciones en las propiedades físicas \\ y mecánicas de la madera de Balanites \\ aegyptiaca según tres orígenes distintos
}

Balanites aegyptiaca (datilero del desierto) es una especie característica y emblemática de las zonas secas de África y Asia. Tiene una gran importancia socioeconómica en su área de distribución natural debido a sus múltiples usos. Sin embargo, no se conocen bien las propiedades tecnológicas de su madera, lo que da lugar a usos a veces inadecuados y que podrían ampliarse. Para adaptar mejor sus aplicaciones a sus propiedades, determinamos los indicadores de estabilidad física (densidad, infradensidad, contracción radial total, contracción tangencial total, contracción volumétrica total y punto de saturación de las fibras) y los indicadores de comportamiento mecánico (módulo de elasticidad longitudinal, tensión de ruptura en flexión y compresión) de esta madera. Se analizaron noventa y tres muestras tomadas de trece árboles de las zonas saheliana y sudanesa chadianas y de la zona saheliana senegalesa. Los resultados obtenidos muestran que para las tres procedencias, la madera de $B$. aegyptiaca es medianamente pesada $\left(797 \mathrm{~kg} / \mathrm{m}^{3}\right)$ con una estabilidad dimensional media: la anisotropía de contracción es superior a 2 (2,2), la contracción radial total y la contracción tangencial total son medias, respectivamente iguales al $4 \%$ y al $8,5 \%$. Sus características mecánicas son medias (tensiones de ruptura en compresión y flexión estática iguales a 49,4 MPa y 104,5 MPa respectivamente) con un bajo módulo de elasticidad longitudinal (de $10473 \mathrm{MPa}$ ). La comparación de los resultados obtenidos según las zonas de muestreo reveló tendencias variables en función de las características estudiadas. Sin embargo, estas variaciones entre los tres orígenes siguen siendo limitadas. Los resultados del estudio demuestran que la madera de $B$. aegyptiaca podría utilizarse adecuadamente como material para una mayor variedad de usos, siempre que se lleve a cabo una gestión adecuada que permita su restauración por plantación.

Palabras clave: Balanites aegyptiaca, madera, propiedades mecánicas, propiedades físicas, origen saheliano, origen sudanés, Senegal, Chad. 


\section{Introduction}

Balanites aegyptiaca, appelé dattier du désert ou " savonnier " au Tchad, est un arbre épineux pouvant atteindre $17 \mathrm{~m}$ de haut pour un diamètre de fût allant jusqu'à $60 \mathrm{~cm}$ (Hall, 1992, 2004). Cette espèce de la famille des Zygophyllaceae, emblématique des zones arides, est très appréciée par les populations locales de toute sa zone naturelle de répartition. Elle répond en effet à une très large gamme d'emplois : usages alimentaires de parties de l'arbre (feuilles, pulpe des fruits, amandes), applications médicinales, fabrication de poisons utilisés pour la pêche, de savon pour la lessive, d'encre, de teinture pour les cuirs (Creac'h, 1940 ; Le Floc'h et Aronson, 2013 ; Dendoncker et al., 2015 ; Adamou et al., 2020). Son aire de répartition est très étendue. L'arbre peut être présent dans des régions dont la pluviométrie varie de 100 à $1000 \mathrm{~mm}$ (Le Floc'h et Aronson, 2013) ; il peut vivre plus de 100 ans (Weber et Montes, 2010). Cette espèce est surtout présente dans les zones arides d'Afrique.

Le dattier du désert a fait l'objet d'études qui portent essentiellement sur l'huile extraite de ses graines, qui présente des propriétés exceptionnelles antioxydantes, anti-inflammatoires et antivirales (Singh et al., 2020 ; Nitiema et al., 2020 ; Khamis et al., 2020 ; Iroha et Hamilton-Amachree, 2019). En revanche, les caractéristiques de son bois ont été très peu étudiées bien qu'il soit très utilisé et apprécié localement du fait de ses propriétés mécaniques, de son haut pouvoir calorifique et de sa bonne résistance aux insectes (Le Floc'h et Aronson, 2013 ; Hall, 2004 ; Creac'h, 1940). Cette espèce est surtout utilisée en construction (Ganaba et al., 2004), pour la fabrication d'outils agricoles (manche de houe, hache...), d'ustensiles de cuisine (mortier, pilon, planche à découper...), de tablettes pour l'écriture des versets coraniques, et comme source d'énergie.

Les quelques études disponibles sur le bois de B. aegyptiaca portent sur sa structure anatomique (Parameswaran et Conrad, 1982), sur la corrélation entre la densité et la croissance des arbres (Weber et Montes, 2010), et sur les relations entre la structure anatomique du bois et ses propriétés physiques et mécaniques (Mohammed, 2019).

Présentant un fort potentiel pour le Sahel du fait de sa tolérance à la sécheresse (Adamou et al., 2020 ; Khamis et al., 2015 ; Andersen et Krzywinski, 2007 ; Gonzalez, 2001 ; Bernus, 1979), le développement de cette essence constitue un enjeu important pour plusieurs pays de cette région qui font face au problème de l'avancée de la désertification. Balanites aegyptiaca a été retenue parmi les quatre espèces d'arbres à utiliser pour le projet de la grande muraille verte d'Afrique, projet initialement conçu comme une zone de plantation de $15 \mathrm{~km}$ de large et d'environ $7800 \mathrm{~km}$ de long, du Sénégal jusqu'à Djibouti en passant par 11 pays, qui est maintenant pensé comme une opportunité pour renforcer la résilience des écosystèmes (Goffner et al., 2019). Compte tenu de son intérêt majeur et des perspectives d'utilisation qu'offre cette espèce, il est apparu important de mieux connaître les propriétés et le comportement technologique de son bois, afin de développer et mieux adapter ses appli- cations à ses caractéristiques, et de favoriser l'adéquation qualité-emploi. Ce bois est déjà très utilisé localement sous forme de bois ronds pour la fabrication artisanale de petits objets, ainsi qu'en construction : grosses branches et troncs de petit diamètre pour la toiture des cases traditionnelles, tronc entier pour leur poteau central.

Afin de répondre aux objectifs de cette étude, nous nous sommes intéressés aux caractéristiques technologiques suivantes et à leurs variations selon les provenances : - indicateurs de stabilité physique du bois : masse volumique à $12 \%$ d'humidité et infradensité, retrait total de séchage (radial, tangentiel et volumique), et point de saturation des fibres. Ces caractéristiques sont des indicateurs majeurs de la qualité du bois et de sa stabilité après mise en œuvre (Guibal et al., 2015 ; Segla et al., 2015 ; Hounlonon et al., 2017) ; pour rappel, le taux d'humidité d'une pièce de bois est le rapport entre la masse d'eau qu'elle contient (différence entre sa masse humide et sa masse anhydre) et sa masse anhydre ;

- indicateurs de comportement mécanique à 12 \% d’humidité : module d'élasticité longitudinal en flexion, contrainte de rupture en flexion statique et contrainte de rupture en compression longitudinale (Guibal et al., 2015 ; Langbour et al., 2008 ; Morel et al., 2017).

\section{Matériels et méthodes}

\section{Échantillonnage et méthode d'obtention des éprouvettes d'essai}

Le matériel végétal a été prélevé dans deux pays sahéliens, le Tchad et le Sénégal (figure 1). L'échantillonnage a été réalisé en tenant compte de l'état sanitaire des arbres (arbres apparaissant bien portants avec un port droit) et de leurs données dendrométriques : hauteur totale et circonférence du tronc (tableau I).

Au Tchad, les échantillons ont été prélevés dans deux zones climatiques différentes, la zone sahélienne (région de N'Djamena avec une pluviométrie moyenne annuelle de $556 \pm 121 \mathrm{~mm}$, et une température de $15^{\circ} \mathrm{C}$ pour la moyenne

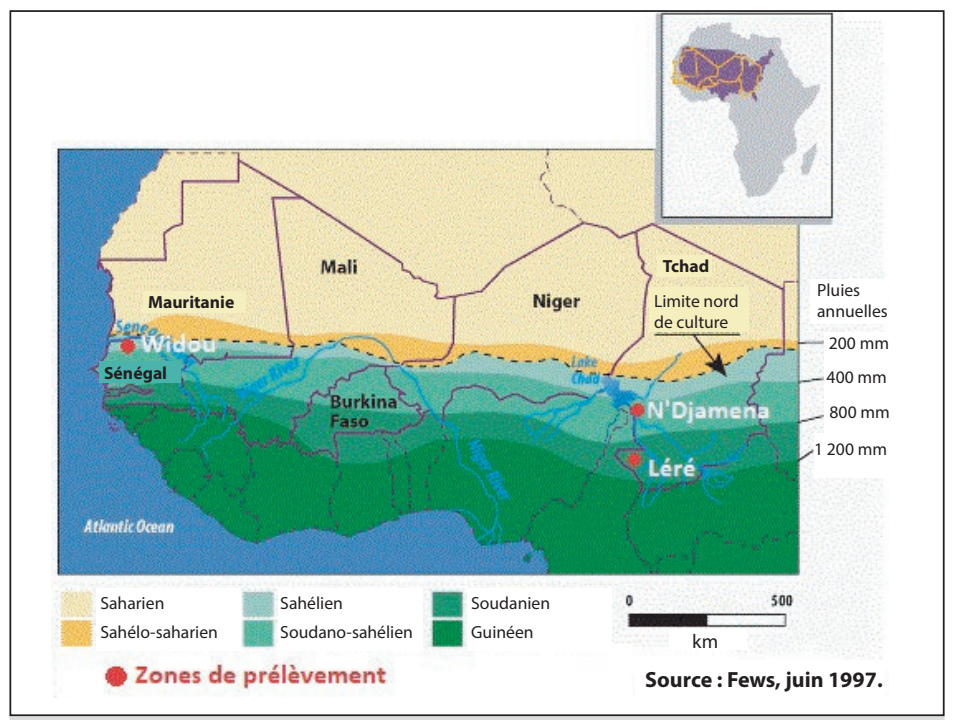

Figure 1.

Zones d'étude.

Study areas. 


\section{Tableau I.}

Coordonnées GPS des prélèvements et données dendrométriques. GPS coordinates of the samples and dendrometric data.

\begin{tabular}{|c|c|c|c|c|c|c|}
\hline Provenance & $N^{\circ}$ pied & $\begin{array}{c}\text { Longitude } \\
\text { (degrés décimaux) }\end{array}$ & $\begin{array}{c}\text { Latitude } \\
\text { (degrés décimaux) }\end{array}$ & $\begin{array}{c}\text { Hauteur } \mathrm{h} \\
\text { du tronc }(\mathrm{cm})\end{array}$ & $\begin{array}{c}\text { Diamètre D } \\
\text { à } \mathrm{h}=1 \mathrm{~m}(\mathrm{~cm})\end{array}$ & $\begin{array}{l}\text { Élancement } \\
\text { du tronc }(h / D)\end{array}$ \\
\hline \multirow{4}{*}{$\begin{array}{l}\text { Tchad zone } \\
\text { sahélienne }\end{array}$} & 1 & 15,11338 & 12,05515 & 162 & 20,6 & 7,9 \\
\hline & 2 & 15,11248 & 12,05421 & 187 & 24,3 & 7,7 \\
\hline & 3 & 15,11191 & 12,05438 & 170 & 27,8 & 6,1 \\
\hline & 4 & 15,11366 & 12,05473 & 167 & 25,4 & 6,6 \\
\hline \multirow{3}{*}{$\begin{array}{l}\text { Tchad zone } \\
\text { soudanienne }\end{array}$} & 1 & 14,08274 & 9,64513 & 179 & 22,8 & 7,9 \\
\hline & 2 & 14,08517 & 9,64718 & 185 & 24,6 & 7,5 \\
\hline & 3 & 14,08312 & 9,64235 & 177 & 25,5 & 6,9 \\
\hline \multirow{6}{*}{$\begin{array}{l}\text { Sénégal zone } \\
\text { sahélienne }\end{array}$} & 1 & $-15,33447$ & 15,9028 & 142 & 34,1 & 4,2 \\
\hline & 2 & $-15,32110$ & 15,91606 & 180 & 28,3 & 6,7 \\
\hline & 3 & $-15,32230$ & 15,93358 & 137 & 31,5 & 4,4 \\
\hline & 4 & $-15,29183$ & 15,98063 & 144 & 31,5 & 4,6 \\
\hline & 5 & $-15,30823$ & 15,99890 & 155 & 25,5 & 6,1 \\
\hline & 6 & $-15,30741$ & 16,05847 & 196 & 28,6 & 6,9 \\
\hline
\end{tabular}

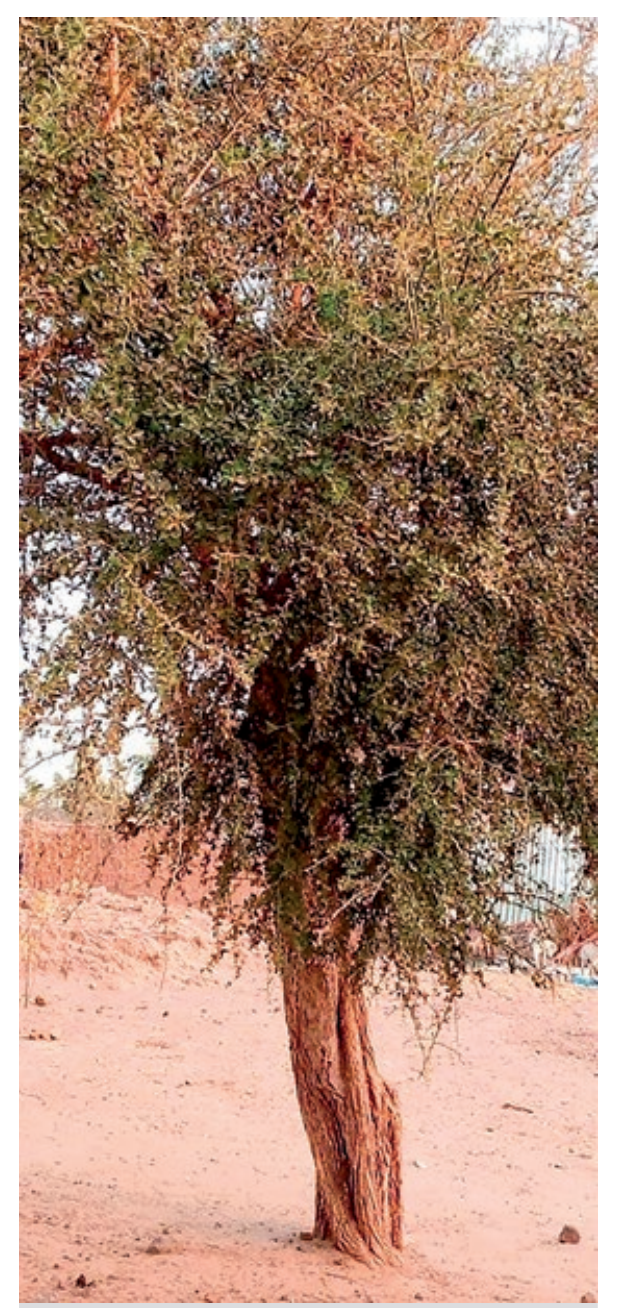

Photo 2.

Arbre $n^{\circ} 2$ (Léré).

Tree No. 2 (Léré).

Photo D. Dougabka. des minimas du mois le plus froid et de $44^{\circ} \mathrm{C}$ pour la moyenne des maximas du mois le plus chaud) et la zone soudanienne (région de Léré avec une pluviométrie annuelle moyenne de $860 \pm 144 \mathrm{~mm}$, et une température moyenne mensuelle allant de 25 à $35^{\circ} \mathrm{C}$, entre 1980 et 2015). En juillet 2018, quatre arbres ont été prélevés dans la région de N'Djamena et trois à Léré, sur des terrains sablo-argileux (photos 1 et 2). Les coordonnées GPS des prélèvements sont données dans le tableau I.

Au Sénégal, les échantillons ont été prélevés en zone sahélienne en avril 2016, plus précisément dans les alentours du village de Widou Thiengoly situé au nord du pays (pluviométrie moyenne annuelle de $298 \pm 87 \mathrm{~mm}$ et température moyenne mensuelle comprise entre 20 à $38^{\circ} \mathrm{C}$, entre 1940 et 2015). L'échantillonnage est constitué de six arbres prélevés sur des sols sablo-argileux. Les coordonnées GPS des prélèvements et les données dendrométriques sont présentées dans le tableau I.

Dans les deux pays et les trois zones de prélèvement, la densité d'arbres à l'hectare est faible, estimée à moins de 5 arbres à l'hectare, et n'influe donc pas sur les caractéristiques physiques et mécaniques du bois.

Les éprouvettes utilisées pour les essais ont été obtenues selon le processus suivant (figure 2 ) :

- chaque arbre a été abattu à $50 \mathrm{~cm}$ au-dessus du sol ; cette hauteur traditionnelle d'abattage facilite l'opération d'abattage manuel à la hache ;

- dans chaque tronc, la première bille d'environ $1 \mathrm{~m}$ de long au-dessus de la découpe a été prélevée (photo 3) ;

- les billes ont été transportées dans une scierie où elles ont été débitées en plateaux diamétraux de $60 \mathrm{~cm}$ de longueur et $6 \mathrm{~cm}$ d'épaisseur à l'aide d'une scie à ruban ;

- les plateaux ont fait l'objet d'un premier séchage naturel de trois mois sous abri sur le site de débit, puis ont été stabilisés au Cirad à Montpellier en chambre climatique à $20^{\circ} \mathrm{C}$ et $65 \%$ d'humidité relative (conditions climatiques permettant une stabilisation du bois à un taux d'humidité théorique de $12 \%$ ) ; 
- après trois semaines de stabilisation des bois, les sections transversales des plateaux ont été marquées pour repérer la position des ébauches d'éprouvettes destinées aux essais ; le marquage a été réalisé de façon à respecter la bonne orientation des sections des éprouvettes suivant la direction radiale et la direction tangentielle ;

- les ébauches (section nominale augmentée d'une surcote) ont été débitées dans les plateaux puis de nouveau stabilisées sous atmosphère contrôlée à $20^{\circ} \mathrm{C}$ et $65 \%$ d'humidité relative jusqu'à obtenir un poids constant ;

- après stabilisation finale, ces ébauches ont été usinées en barreaux de $20 \times 20 \mathrm{~mm}^{2}$ de section (dimension radiale $\mathrm{x}$ dimension tangentielle) et de longueur égale à la longueur du plateau; dans ces barreaux, trois groupes d'éprouvettes de même section $20 \times 20 \mathrm{~mm}^{2}$ mais de longueurs $L$ différentes ont été prélevés en fonction du type d'essai à réaliser : $L=360 \mathrm{~mm}$ pour les essais de flexion (contrainte de rupture et module d'élasticité longitudinal) ; $L=60 \mathrm{~mm}$ pour les essais de compression ; $L=10 \mathrm{~mm}$ pour les propriétés physiques.

Tous les essais ont été réalisés au Cirad à Montpellier.

\section{Méthodes d'essai}

\section{Propriétés physiques}

Les propriétés physiques de référence étudiées sont les suivantes : masse volumique à $12 \%$, infradensité, retrait radial total de séchage, retrait tangentiel total de séchage, retrait volumique total, point de saturation des fibres et anisotropie de retrait déduite des retraits linéaires (Guibal et al., 2015).

La masse volumique à $12 \%$ est déterminée simultanément au module d'élasticité longitudinal. La méthode de mesure correspondante est décrite dans « Propriétés mécaniques » ci-après.

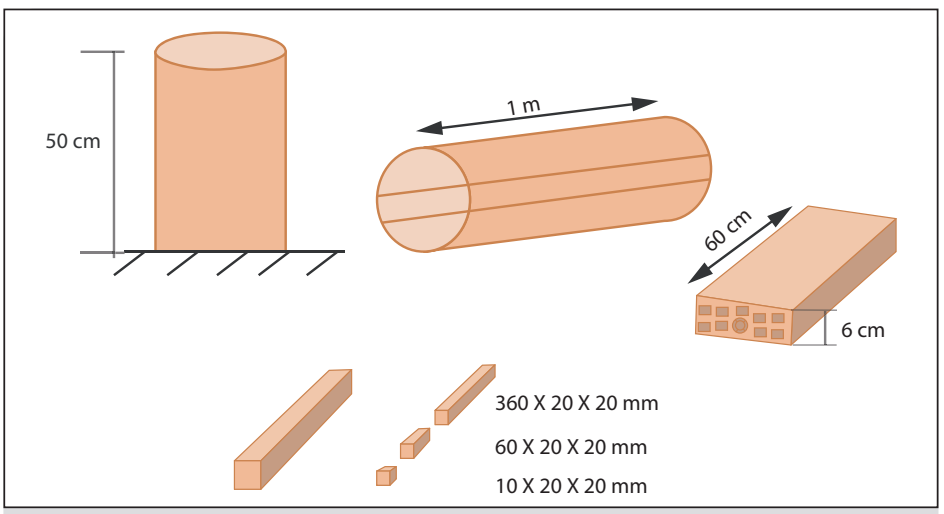

Figure 2.

Processus d'obtention des éprouvettes.

The process of obtaining the specimens.

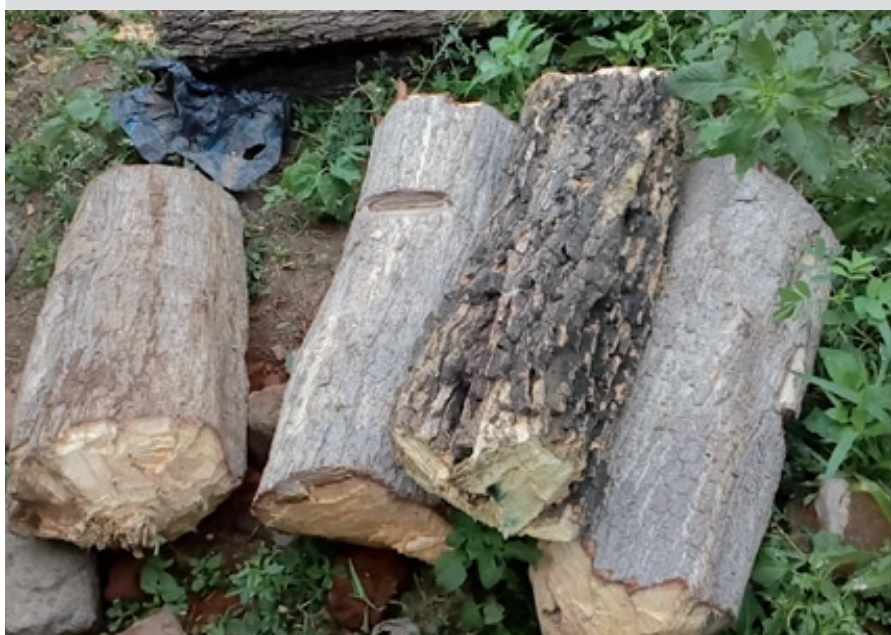

Photo 3.

Billons prélevés après abattage.

Bucked wooden log after felling.

Photo D. Dougabka.

Les cinq autres propriétés physiques sont déterminées sur les mêmes éprouvettes suivant un protocole unique.

Ces éprouvettes ont été, dans un premier temps, saturées dans de l'eau distillée pendant trois jours puis soumises à trois cycles de vide-pression dans un autoclave afin de chasser d'éventuelles poches d'air piégées dans le bois.

Après saturation, les éprouvettes ont été pesées et leurs dimensions radiale et tangentielle mesurées.

Les appareils utilisés pour ces mesures, ainsi que pour les mesures suivantes, sont les suivants : une balance de marque Sartorius de précision 0,001 g (photo 4) ; un comparateur de marque Mitutoyo de précision 0,001 mm (photo 5).

Le volume saturé des éprouvettes a été déterminé par la méthode de la double pesée (utilisation du principe de la poussée d'Archimède) : ce volume est déterminé par la masse du volume d'eau déplacé dans un bécher lors de l'immersion d'une éprouvette dans ce bécher rempli d'eau et pesé avant puis après l'immersion.

Après cette première étape de mesures à l'état saturé, et en vue de déterminer le point de saturation des fibres (PSF), les éprouvettes ont été stabilisées en enceinte climatique successivement à $85 \%$ d'humidité relative de l'air et $30^{\circ} \mathrm{C}$ (équilibre hygroscopique du bois voisin de $18 \%$ ), $65 \%$ d'humidité relative de l'air et $20^{\circ} \mathrm{C}$ (équilibre hygroscopique voisin de $12 \%$ ) puis $30 \%$ d'humidité relative de l'air et $20^{\circ} \mathrm{C}$ (équilibre hygroscopique voisin de $6 \%$ ).

Elles ont été enfin stabilisées jusqu'à l'état anhydre en étuve sèche à $103^{\circ} \mathrm{C}$. Selon la norme française NFEN 13183-1 de juin 2002, un échantillon de bois est considéré comme anhydre lorsque la variation de sa masse entre deux pesées successives dans un intervalle de 2 heures ne dépasse pas $0,1 \%$.

À chaque état stabilisé, la masse et les dimensions radiale et tangentielle des éprouvettes ont été mesurées. Ces mesures ont permis de déterminer les cinq propriétés étudiées et les deux propriétés déduites. 


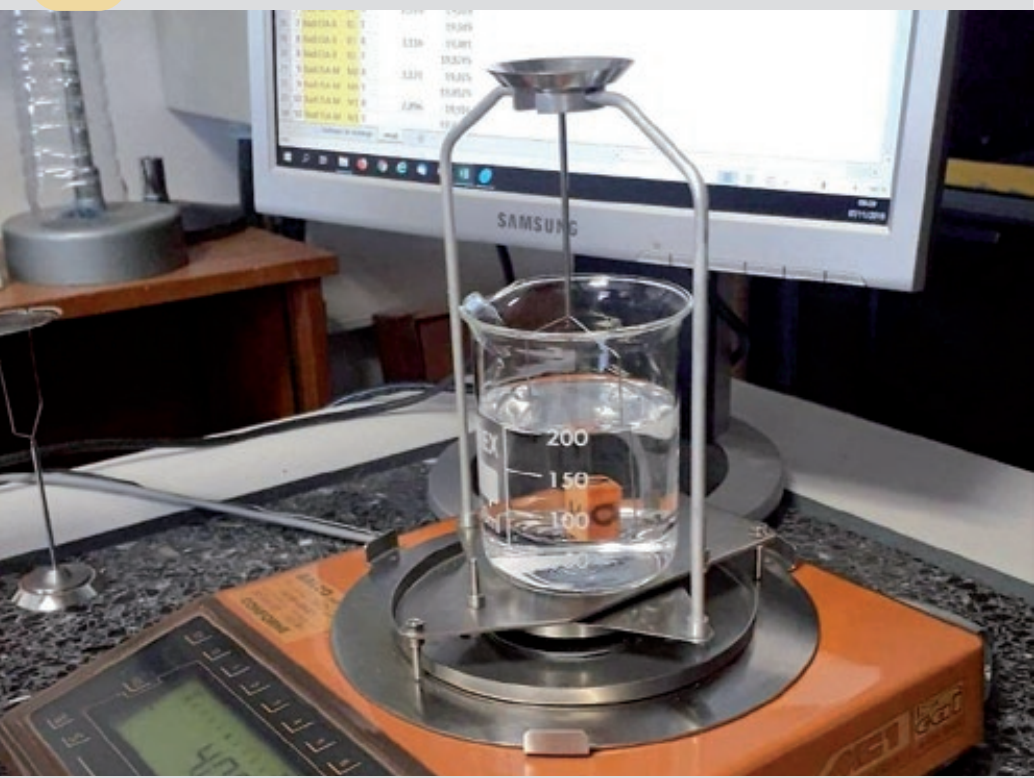

Photo 4.

Balance Sartorius.

Sartorius scale device.

Photo D. Dougabka.

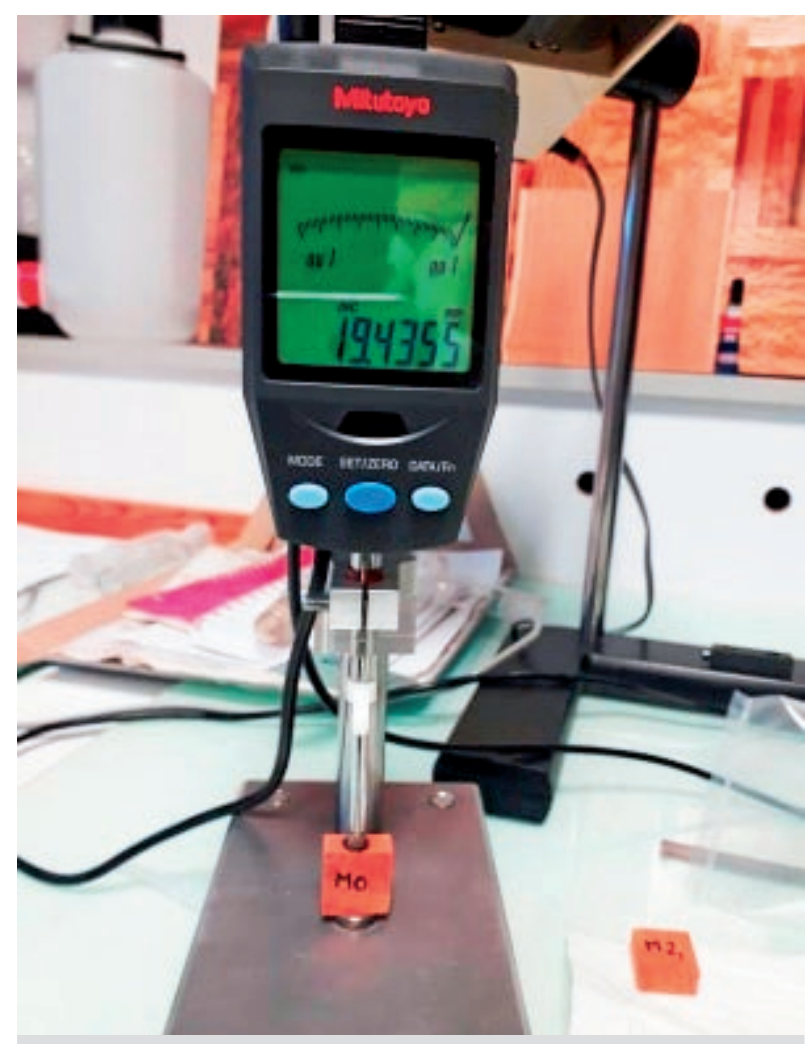

Photo 5.

Comparateur Mitutoyo.

Mitutoyo dial gauge.

Photo D. Dougabka.

\section{Infradensité}

L'infradensité « d » d'un échantillon est le rapport entre sa masse anhydre Ma et son volume saturé Vs. Elle s'exprime en $\mathrm{kg} / \mathrm{m}^{3}$. Cette caractéristique de référence présente l'avantage d'être facile à déterminer lorsque l'on ne dispose pas d'enceinte climatique pour stabiliser des échantillons à des conditions de température et d'humidité de l'air contrôlées. Elle permet aussi de calculer la biomasse anhydre du bois des troncs à partir du volume sous écorce mesuré lors d'inventaires forestiers.

$d=\frac{M_{a}}{V_{s}}$ (équation 1)

\section{Retraits linéaires transversaux totaux}

Les retraits linéaires transversaux de séchage constituent des indicateurs de la variation dimensionnelle du bois quand son humidité diminue en dessous du point de saturation des fibres. Cette variation dimensionnelle est différente suivant les directions tangentielle et radiale du bois. Ces retraits s'expriment en pourcentage et sont calculés à l'aide de la formule suivante (norme NF B 51-006, septembre 1985) :

$\mathrm{R}_{\mathrm{T}, \mathrm{R}}=\frac{\mathrm{e}_{\mathrm{s}}-\mathrm{e}_{\mathrm{o}}}{\mathrm{e}_{\mathrm{s}}} \times 100$ (équation 2)

où $R_{T, R}$ sont les retraits tangentiels et radiaux, $R_{T}$ pour la direction tangentielle et $R_{R}$ pour la direction radiale, $e_{s}$ et $e_{0}$ respectivement les dimensions des échantillons à l'état saturé et anhydre suivant la direction considérée (radiale ou tangentielle).

Quelle que soit l'essence de bois considérée, on a toujours $R_{T}>R_{R}$.

\section{Anisotropie de retrait}

L'anisotropie du retrait est déterminée par le rapport entre le retrait tangentiel et le retrait radial.

Ce paramètre constitue un bon indicateur du niveau des déformations subies par une pièce de bois soumise à des variations d'humidité, et de la stabilité d'un bois durant son séchage et sa mise en service (Gérard et al., 2016).

Lorsque ce rapport $R_{T} \div R_{R}$ est supérieur à 2, on estime qu'un bois risque d'être instable en service et sujet aux fentes et aux déformations.

\section{Retrait volumique total}

Le retrait volumique total de séchage $R_{B}$ correspond à la variation de volume d'une pièce de bois entre l'état saturé et l'état anhydre.

Le retrait longitudinal étant le plus souvent très faible par rapport aux retraits linéaires transversaux, la somme du retrait radial et du retrait tangentiel $\left(R_{R}+R_{T}\right)$ constitue une bonne approximation du retrait volumique.

$\mathrm{R}_{\mathrm{B}}=\frac{\mathrm{V}_{\mathrm{s}}-\mathrm{V}_{\mathrm{a}}}{\mathrm{V}_{\mathrm{s}}} \times 100 \approx \mathrm{R}_{\mathrm{R}}+\mathrm{R}_{\mathrm{T}}$ (équation 3)

avec Vs = volume saturé et Va = volume anhydre. 


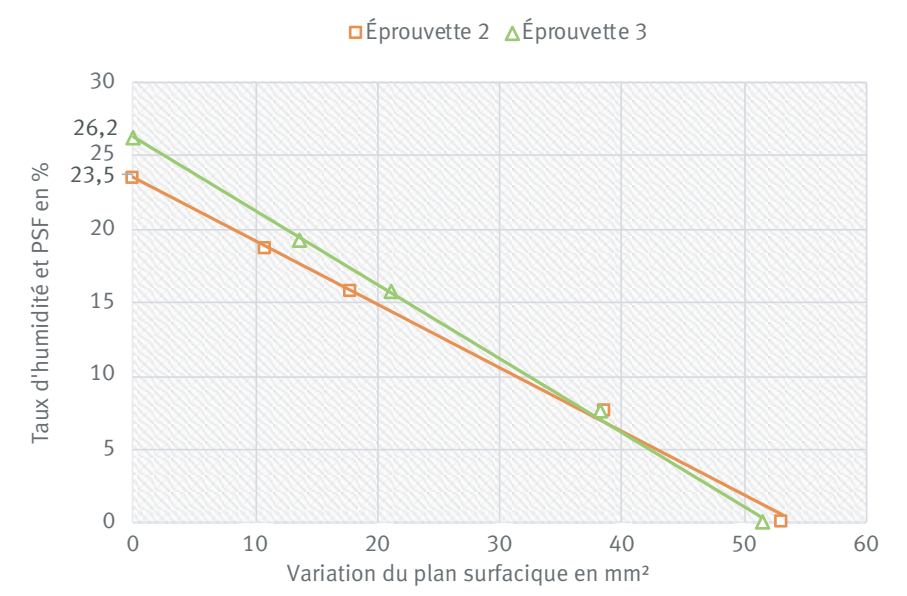

Figure 3.

Exemple de détermination du PSF de deux éprouvettes de Balanites aegyptiaca par calcul de l'ordonnée à l'origine des courbes [Taux d'humidité] = $\mathrm{f}$ [Variations surfaciques]. Example of the determination of the PSF of two Balanites aegyptiaca specimens by calculating the intercept of the curves [Moisture content $=$ f [Areal variations].

\section{Point de saturation des fibres}

Le point de saturation des fibres (PSF) est le taux d'humidité du bois saturé en eau liée, taux en dessous duquel les dimensions du bois varient en fonction de l'humidité ambiante.

Il est compris le plus souvent entre $20 \%$ et $40 \%$ suivant les essences, mais se situe généralement entre $25 \%$ et 30 \% (Gérard et al., 2016).

Il est obtenu en stabilisant les échantillons de bois à différents taux d'humidité intermédiaires entre l'état saturé et l'état anhydre, à des conditions de température et d'humidité relative de l'air correspondant à des taux d'humidité théorique du bois successivement proches de $18 \%, 12 \%$ et $6 \%$. À chaque étape, la masse et les dimensions des éprouvettes sont mesurées. Ces dimensions permettent de déterminer les surfaces transversales correspondant au taux d'humidité de stabilité (exemple : surface transversale à $18 \%$ d'humidité = dimension radiale à $18 \%$ × dimension tangentielle à $18 \%$ ). Le taux d'humidité exact des échantillons est déterminé en fin d'expérimentation après leur pesée à l'état anhydre.

Le PSF est déterminé par l'ordonnée à l'origine des droites de régression entre les variations de surface transversale des échantillons et leur taux d'humidité (figure 3) : [taux d'humidité] = f [variations surfaciques]. Il correspond au taux humidité au-delà duquel les variations dimensionnelles sont nulles (Kokutse et al., 2010). La surface transversale est le produit de la dimension radiale et de la dimension tangentielle de la section transversale de l'éprouvette.

Le PSF du bois est considéré comme faible pour des valeurs inférieures à $25 \%$, moyen pour des valeurs comprises entre $25 \%$ et $35 \%$, et élevé pour des valeurs supérieures à 35 \% (Gérard et al., 2016).

\section{Propriétés mécaniques}

Les propriétés mécaniques de référence étudiées sont le module d'élasticité longitudinal, la contrainte de rupture en flexion statique et la contrainte de rupture en compression longitudinale.

\section{Mesure du module d'élasticité longitudinal et de la masse volumique par méthode vibratoire}

Le module d'élasticité longitudinal du bois détermine son aptitude à se déformer de manière élastique, c'est-àdire de manière réversible. Il est établi de façon normalisée sur les bois stabilisés en chambre climatique à $20^{\circ} \mathrm{C}$ et $65 \%$ d'humidité relative de l'air, soit une humidité théorique du bois de $12 \%$. Cette propriété caractérise la proportionnalité entre la charge et la déformation. C'est un indicateur de la rigidité du bois.

La méthode de mesure utilisée met en œuvre le dispositif BING@ (Beam Identification by Nondestructive Grading). Cette méthode non destructive a été développée par le Cirad. Elle repose sur l'étude des vibrations d'une pièce de bois ou de tout autre matériau. Le dispositif permet de déterminer les caractéristiques élastiques (module d'élasticité longitudinal, module de cisaillement transverse) de tout matériau rigide. La mesure nécessite une éprouvette élancée, de géométrie régulière, sans défaut, et dont les conditions d'appui sont maîtrisées. L'éprouvette est soumise à des vibrations longitudinales ou transversales dont l'enregistrement et l'analyse conduisent à la détermination des caractéristiques recherchées.

Le principe de la mesure consiste à analyser les fréquences propres de résonance obtenues après une excitation impulsionnelle sur l'une des extrémités d'une éprouvette reposant sur deux bracelets élastiques de faible rigidité (Bordonné, 1989).

Les paramètres ainsi mesurés à l'aide du dispositif sont la densité du bois sec déterminée par la pesée de l'échantillon et la mesure de ses trois dimensions, le module spécifique déduit des fréquences propres (en mètres carrés par seconde carrée, $\mathrm{m}^{2} / \mathrm{s}^{2}$ ), et le rapport entre le module d'élasticité longitudinal et le module de cisaillement. Le module d'élasticité longitudinal est égal au produit de la densité par le module spécifique.

Le dispositif d'analyse est composé d'une balance, d'un support-échantillon, d'un microphone, d'un filtre amplificateur, d'un convertisseur de signal analogique en signal numérique, d'un tube de sollicitation à bille ou d'un maillet, et d'un ordinateur pour l'acquisition et le traitement des données par transformée de Fourrier rapide (photo 6).

Les mesures ont été réalisées sur des éprouvettes de $360 \times 20 \times 20 \mathrm{~mm}^{3}$ stabilisées en conditions standards à $20{ }^{\circ} \mathrm{C}$ et $65 \%$ d'humidité relative de l'air, soit une humidité théorique du bois de $12 \%$. L'espacement entre les deux bracelets élastiques est d'environ la moitié de la longueur de l'éprouvette ; la précision de cet écartement est sans impact sur la mesure (Bordonné, 1989). 


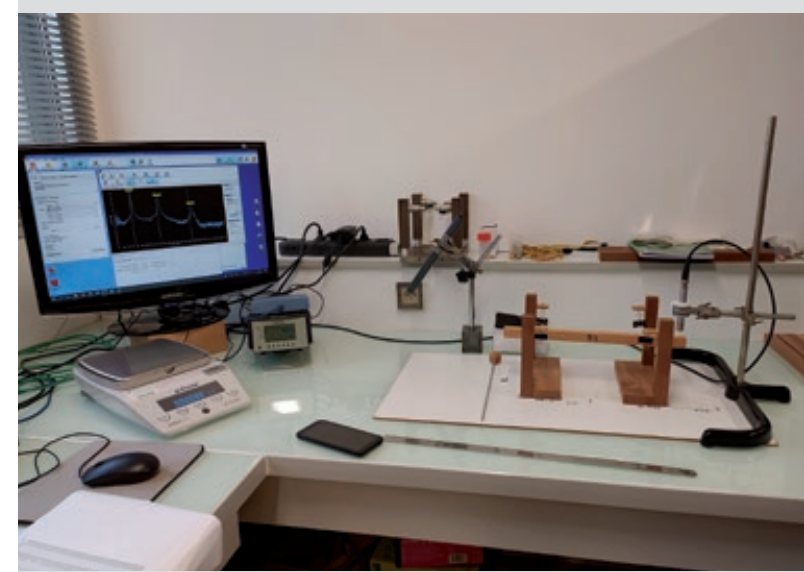

Photo 6.

Dispositif du test BING@.

BING (c) test device.

Photo D. Dougabka.

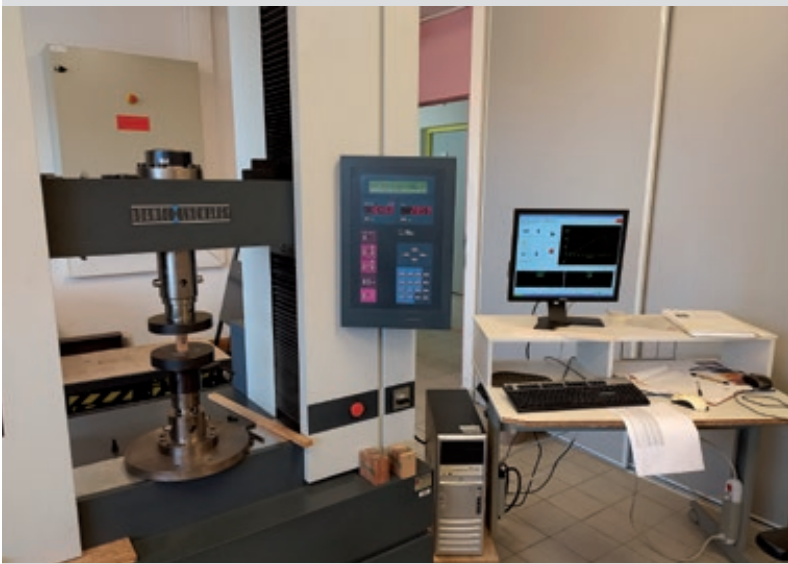

Photo 7.

Banc d'essais de compression.

Compression test bench.

Photo D. Dougabka.

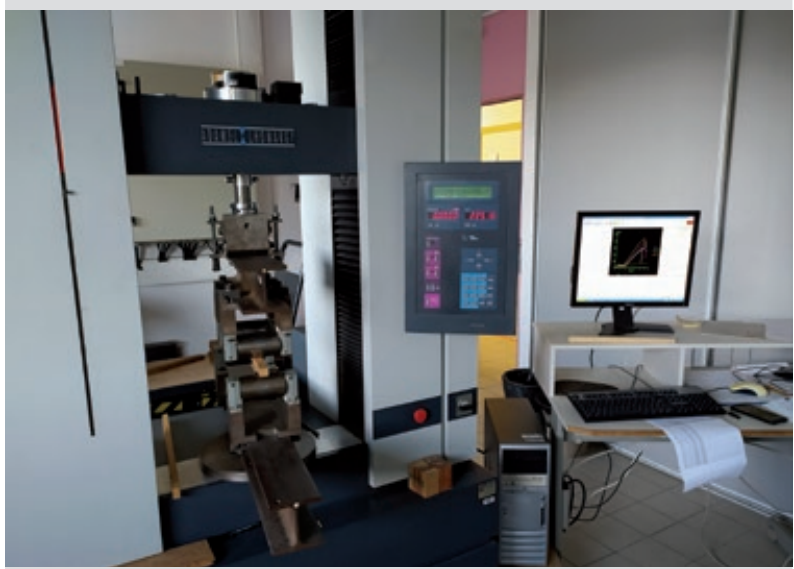

Photo 8.

Banc d'essais de flexion.

Bending test bench.

Photo D. Dougabka.

\section{Contrainte de rupture en compression longitudinale}

La contrainte de rupture en compression longitudinale correspond à la contrainte qu'il est nécessaire d'appliquer suivant la direction parallèle au fil du bois pour obtenir la rupture d'une éprouvette parallélépipédique ayant la forme d'un barreau et orientée longitudinalement.

Cette caractéristique a été déterminée selon la méthode de mesure définie par la norme NF ISO 13061-17 (juin 2018). Les mesures sont effectuées sur des éprouvettes normalisées orientées longitudinalement, de dimensions $60 \times 20 \times 20 \mathrm{~mm}^{3}$, et stabilisées en conditions standards. Les essais ont été réalisés sur une machine universelle de marque Adamel (photo 7) équipée d'une cellule de charge maximale de $100 \mathrm{kN}$. La machine est constituée d'un plateau rotulé fixe sur lequel l'échantillon est posé et d'une cellule de charge permettant d'appliquer la force de compression à l'échantillon. La vitesse de réalisation des essais est égale à $0,01 \mathrm{~mm} / \mathrm{s}$. L'acquisition des données (charge, déplacement) est faite à l'aide d'un ordinateur. La contrainte de rupture est égale au rapport entre la force appliquée et la section de l'éprouvette :

$\sigma=\frac{\mathrm{F}}{A} \quad$ (équation 4)

où $\sigma$ est la contrainte de rupture en compression (MPa), $\mathrm{F}$ est la charge à la rupture ( $\mathrm{N})$, et $\mathrm{A}$ est la section de l'éprouvette $\left(\mathrm{mm}^{2}\right)$.

\section{Contrainte de rupture en flexion statique}

Cette caractéristique correspond à la contrainte qu'il est nécessaire d'appliquer par deux appuis dans la zone centrale d'une éprouvette parallélépipédique ayant la forme d'un barreau reposant sur deux appuis pour atteindre sa rupture après chargement progressif (flexion 4 points). Elle est déterminée sur des bois stabilisés en conditions standards, et dans notre cas suivant la norme française NF B 51-008 (26 juillet 2017). L'essai a été réalisé sur les éprouvettes de dimensions $360 \times 20 \times 20 \mathrm{~mm}^{3}$ utilisées précédemment pour le module d'élasticité longitudinal. La machine utilisée est la même que celle pour les essais de compression, en adaptant le bâti (photo 8). La durée de l'essai est de 1,5 $\pm 0,5 \mathrm{~min}$. La contrainte maximale est déterminée suivant la formule ci-dessous :

$\sigma=\frac{3 P(l-a)}{2 b h^{2}}$ (équation 5)

où $\sigma$ est la contrainte de rupture en flexion (MPa), $\mathrm{P}$ est la charge totale de rupture $(\mathrm{N})$, I est la distance entre les rouleaux du bâti $(320 \mathrm{~mm})$, a est la distance entre les rouleaux supérieurs $(160 \mathrm{~mm}), \mathrm{b}$ est la largeur de l'éprouvette $(\mathrm{mm})$, et $\mathrm{h}$ est la hauteur de l'éprouvette $(\mathrm{mm})$.

À l'issue de l'essai, le taux d'humidité des éprouvettes est déterminé suivant le protocole défini dans la norme NF EN 13183-1 (juin 2002). 


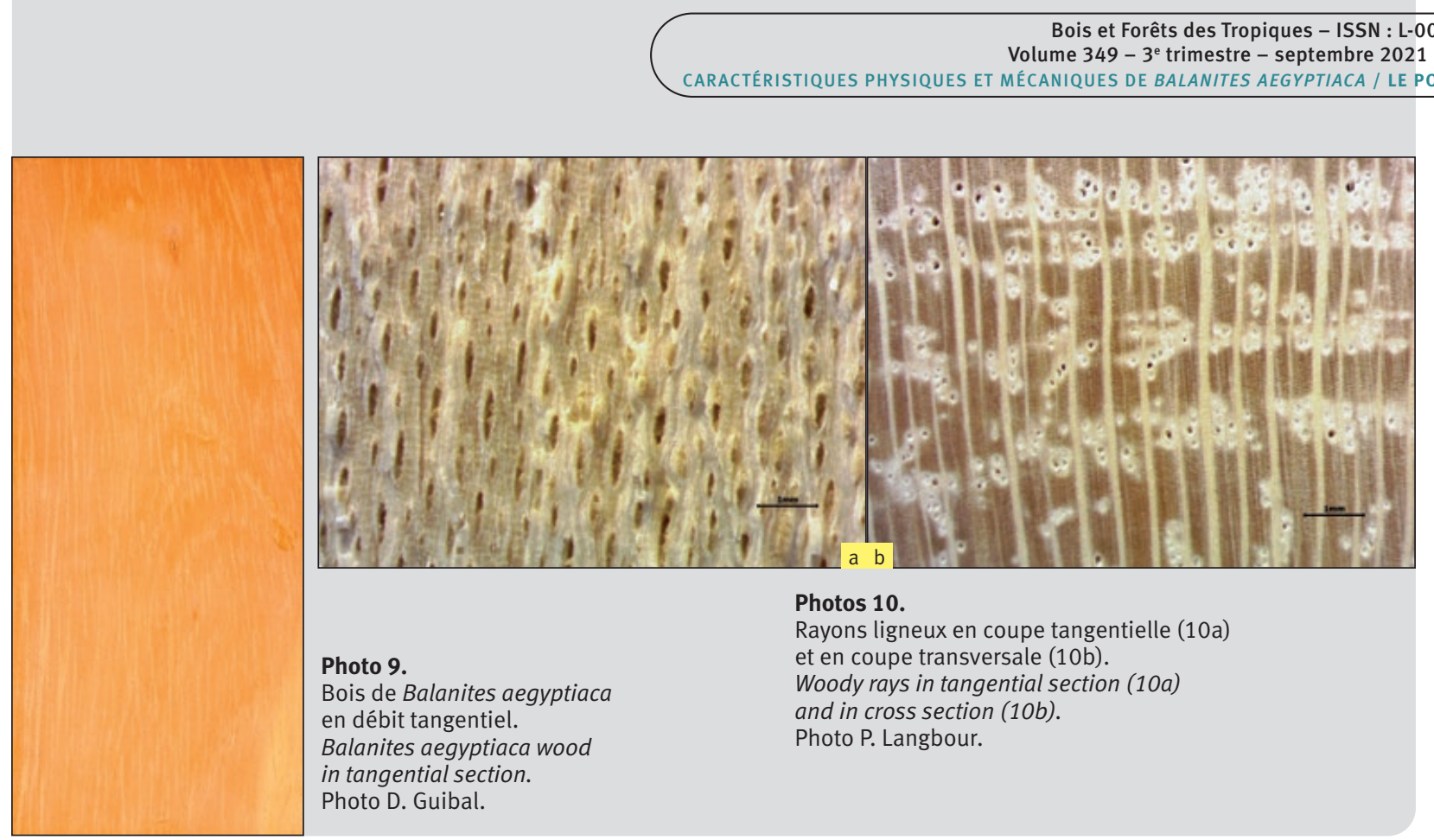

\section{Résultats et discussion}

\section{Descriptif des bois}

L'écorce des arbres exploités est crevassée, très écailleuse, et de couleur beige à brun-gris, relativement mince, jusqu'à 15 mm d'épaisseur. Lors de l'abattage, le tronc n'exsude pas de sève ni de résine particulière. Le bois est à pores diffus, de couleur jaune pâle à brun très clair pouvant virer au grisâtre en cas d'échauffures. L'aubier est indifférencié. Le fil du bois est légèrement ondulé (photo 9). Des cernes de croissance sont bien visibles, d'épaisseur très variable, entre 2 et $9 \mathrm{~mm}$. Les rayons ligneux sont bien visibles, de 120 à $130 \mu \mathrm{m}$ de large et 1 à $2 \mathrm{~mm}$ de hauteur, soit un rapport moyen largeur / hauteur égal à 0,09 (photos 10a et $10 \mathrm{~b}$ ). Le bois frais ne présente pas d'odeur très marquée. Lors du tronçonnage puis par la suite lors du débit à la scie à ruban, le bois apparaît relativement pelucheux. Le cœur des troncs est relativement bien centré ; a priori, le caractère pelucheux du bois n'est donc pas spécifiquement indicateur de bois de tension. Le sciage est cependant facile.

\section{Statistiques descriptives des propriétés étudiées}

Le tableau II présente le résultat des statistiques descriptives des résultats d'essais conduits sur un total de 279 éprouvettes, soit 93 pour chacun des trois formats, obtenues à partir des 13 arbres des trois provenances d'étude (zone sahélienne tchadienne, zone soudanienne tchadienne, zone sahélienne sénégalaise). Les éprouvettes sont sans défaut (fil droit, absence de nœud). L'aubier du bois de $B$. aegyptiaca étant indifférencié, les barreaux ont été prélevés à des distances variables du cœur. Le logiciel utilisé pour le traitement des résultats est XLSTAT 2020.5.1.

\section{Résultats généraux}

Pour les trois provenances confondues, les retraits radial et tangentiel totaux ont une moyenne respective de $4 \%$ et $8,5 \%$, avec une dispersion des résultats plus de deux fois plus élevée pour le retrait radial que pour le retrait tangentiel (coefficient de variation respectivement de $23,1 \%$ et $10,4 \%$ ).

L'anisotropie de retrait moyenne est de 2,2, avec un coefficient de variation de $17,8 \%$, soit une dispersion élevée liée à la dispersion élevée du retrait radial.

Le point de saturation des fibres moyen est de $24 \%$, avec un faible coefficient de variation (6,4 \%) bien que les résultats soient la moyenne des trois provenances.

L'infradensité et la masse volumique ont des moyennes respectives de $652 \mathrm{~kg} / \mathrm{m}^{3}$ et $797 \mathrm{~kg} / \mathrm{m}^{3}$, avec une faible dispersion des résultats (coefficient de variation respectivement de $6,8 \%$ et 5,4\%), ceci bien que, comme pour le PSF, les résultats soient la moyenne des trois provenances.

La contrainte de rupture en compression, en flexion statique, et le module d'élasticité longitudinal ont des moyennes respectives de 49,4 $\mathrm{MPa}, 104,5 \mathrm{MPa}$ et $10473 \mathrm{MPa}$, et des coefficients de variation respectivement de $13,7 \%, 12,4 \%$ et $16,1 \%$, indicateurs d'une dispersion relativement élevée des résultats.

\section{Influence des provenances sur les propriétés étudiées}

Les échantillons ont été prélevés sur des sites géographiquement différents associés à des conditions de croissance très différentes liées à des climats différents, ce qui pourrait expliquer la forte dispersion des résultats, associée à un coefficient de variation élevé. Cette tendance est cependant infirmée pour trois caractéristiques, le point de saturation des fibres, l'infradensité et la masse volumique, dont les résultats sont globalement peu dispersés. 
Tableau II.

Caractéristiques physiques et mécaniques de Balanites aegyptiaca des provenances sahélienne et soudanienne tchadienne et sahélienne sénégalaise.

Physical and mechanical characteristics of Balanites aegyptiaca from the Chadian and Senegalese Sahelian regions.

\begin{tabular}{|c|c|c|c|c|c|c|c|c|c|c|c|c|c|}
\hline $\begin{array}{l}\text { Provenance } \\
\text { (nombre } \\
\text { d'éprouvettes) }\end{array}$ & Statistique & $\begin{array}{c}\mathrm{H} \\
(\%)\end{array}$ & $\begin{array}{l}\mathrm{Rr} \\
(\%)\end{array}$ & $\begin{array}{c}\mathrm{Rt} \\
(\%)\end{array}$ & $\begin{array}{l}\mathrm{Rv} \\
(\%)\end{array}$ & Anis & $\begin{array}{l}\text { PSF } \\
(\%)\end{array}$ & $\begin{array}{c}\text { ID } \\
\left(\mathrm{kg} / \mathrm{m}^{3}\right)\end{array}$ & $\begin{array}{c}M v \\
\left(\mathrm{~kg} / \mathrm{m}^{3}\right)\end{array}$ & $\begin{array}{c}\mathrm{C}_{12} \\
(\mathrm{MPa})\end{array}$ & $\begin{array}{c}\mathrm{F}_{12} \\
(\mathrm{MPa})\end{array}$ & $\begin{array}{c}\mathrm{E}_{12} \\
(\mathrm{MPa})\end{array}$ & $\begin{array}{c}\text { MS } \\
\left(\mathrm{Mm}^{2} / \mathrm{s}^{2}\right.\end{array}$ \\
\hline \multirow{3}{*}{$\begin{array}{l}\text { Résultats pour les } \\
\text { trois provenances } \\
(279)\end{array}$} & Moyenne & 11,5 & 4,0 & 8,5 & 12,4 & 2,2 & 24,0 & 652 & 797 & 49,4 & 104,5 & 10473 & 13,3 \\
\hline & Écart-type & 1,3 & 0,9 & 0,9 & 1,1 & 0,4 & 1,5 & 44 & 41 & 6,8 & 13,0 & 1682 & 2,6 \\
\hline & CV & 11,7 & 23,1 & 10,7 & 9,0 & 17,8 & 6,4 & 6,8 & 5,2 & 13,7 & 12,4 & 16,1 & 19,9 \\
\hline \multirow{3}{*}{$\begin{array}{l}\text { Tchad, zone } \\
\text { soudanienne ( } 51 \text { ) }\end{array}$} & Moyenne & 12,8 & 3,3 & 9,3 & 12,5 & 2,6 & 24,5 & 655 & 814 & 40,9 & 100,1 & 9182 & 11,5 \\
\hline & Écart-type & 0,3 & 0,9 & 0,4 & 0,9 & 0,4 & 1,6 & 28 & 22 & 5,0 & 17,1 & 1650 & 2 \\
\hline & CV & 2 & 26,4 & 4,8 & 7,5 & 16,4 & 6,7 & 4,3 & 2,7 & 12,3 & 17,1 & 18,0 & 17,8 \\
\hline \multirow{3}{*}{$\begin{array}{l}\text { Tchad, zone } \\
\text { sahélienne (60) }\end{array}$} & Moyenne & 12,3 & 3,5 & 8,1 & 11,7 & 2,4 & 22,7 & 646 & 784 & 50,1 & 100,7 & 11493 & 14,7 \\
\hline & Ecart-type & 0,4 & 0,3 & 0,7 & 0,8 & 0,2 & 0,7 & 10 & 10 & 1,9 & 5,0 & 722 & 2,1 \\
\hline & CV & 3 & 8,2 & 8,4 & 6,6 & 10,5 & 3,0 & 1,6 & 1,3 & 3,8 & 4,9 & 6,3 & 14 \\
\hline \multirow{3}{*}{$\begin{array}{l}\text { Sénégal, zone } \\
\text { sahélienne (168) }\end{array}$} & Moyenne & 9,9 & 4,5 & 8,3 & 12,7 & 2,0 & 24,2 & 653 & 793 & 52,0 & 110,1 & 10897 & 13,6 \\
\hline & Écart-type & 0,3 & 0,8 & 0,9 & 1,2 & 0,3 & 1,6 & 56 & 60 & 6,1 & 11,7 & 1534 & 2,8 \\
\hline & CV & 3 & 18,1 & 11,1 & 9,4 & 13,8 & 6,8 & 8,6 & 7,6 & 11,8 & 10,7 & 14,1 & 20,5 \\
\hline
\end{tabular}

$\mathrm{CV}$ : coefficient de variation ; $\mathrm{H}$ : taux d'humidité de stabilisation à $20^{\circ} \mathrm{C}$ et $65 \%$ d'humidité relative de l'air ; Rr : retrait radial total ; Rt : retrait tangentiel total ; Rv : retrait volumique total ; Anis : anisotropie de retrait ; PSF : point de saturation des fibres ; ID : infradensité ; Mv : masse volumique à $12 \% ; C_{12}$ : contrainte de rupture en compression longitudinale à $12 \%$ d'humidité ; $F_{12}$ : contrainte de rupture en flexion statique à $12 \%$ d'humidité ; $\mathrm{E}_{12}$ : module d’élasticité longitudinal à $12 \%$ d'humidité ; MS : module spécifique (= $\mathrm{E}_{12} /$ Mv).

L'influence des provenances sur les propriétés étudiées a été analysée en effectuant un test non paramétrique de Kruskal-Wallis.

Les résultats de ces tests ont été associés à une représentation graphique des distributions sous forme de box plots (figure 4).

La figure 4 montre ainsi que le retrait radial total est significativement plus élevé dans la zone sahélienne sénégalaise $(4,5 \%)$ que dans les deux provenances tchadiennes $(3,3 \%$ et $3,5 \%$; figure $4 a)$.

La différence de retrait tangentiel entre les zones sahéliennes tchadienne et sénégalaise est non significative (figure 4b), mais elle est significativement plus élevée dans la zone soudanienne tchadienne que dans les deux zones sahéliennes tchadienne et sénégalaise (respectivement $9,3 \%$ contre $8,1 \%$ et $8,3 \%$ ).

Cette tendance est l'inverse de celle observée pour les retraits radiaux totaux en termes d'effet provenances.

On n'observe aucune différence significative de retrait volumique entre les trois provenances (figure $4 c$ ). Ce résultat est lié au mode de calcul du retrait volumique en fonction des deux retraits linéaires, et à l'annulation des deux tendances inverses observées sur ces deux retraits.

L'anisotropie de retrait est significativement plus élevée sur les deux provenances tchadiennes (zone soudanienne et zone sahélienne) comparées à la zone sahélienne sénégalaise (figure $4 \mathrm{~d}$ ).

Le point de saturation des fibres dans la zone sahélienne tchadienne est significativement moins élevé que les valeurs obtenues en zone soudanienne tchadienne et en zone sahélienne sénégalaise (figure 4e)
Pour l'infradensité (figure 4f), aucune différence significative n'est observée entre les trois provenances.

Contrairement à l'infradensité, la masse volumique à $12 \%$ est significativement plus élevée en zone soudanienne tchadienne qu'en zone sahélienne tchadienne et sénégalaise ; aucune différence significative n'est observée entre les deux zones sahéliennes (figure 4g).

Des trois caractéristiques mécaniques étudiées, la contrainte de rupture en compression (figure 4h) et le module d'élasticité longitudinal (figure 4j) présentent la même tendance : les valeurs obtenues en zone soudanienne tchadienne sont significativement moins élevées que celles de la zone sahélienne tchadienne et sénégalaise.

Pour la contrainte de rupture en flexion (figure 4i), aucune différence significative n'est observée entre les provenances.

La tendance observée pour le module d'élasticité longitudinal, la contrainte de rupture en flexion et la contrainte de rupture en compression est l'inverse de celle observée pour la masse volumique et l'infradensité : on constate que dans la zone d'étude, où la masse volumique et l'infradensité sont les plus élevées (Tchad, zone soudanienne), le module d'élasticité longitudinal et les contraintes de rupture en flexion et en compression sont les plus faibles, ce qui est contraire aux tendances observées en général. Ce résultat pourrait être lié à un taux très variable des extractibles dans le bois de $B$. aegyptiaca en relation avec la provenance.

Les variations du module spécifique, rapport entre le module d'élasticité longitudinal et la masse volumique, ont été analysées (figure 4h) car cette variable traduit souvent une adaptation aux conditions de croissance (Thibaut, 2016). 


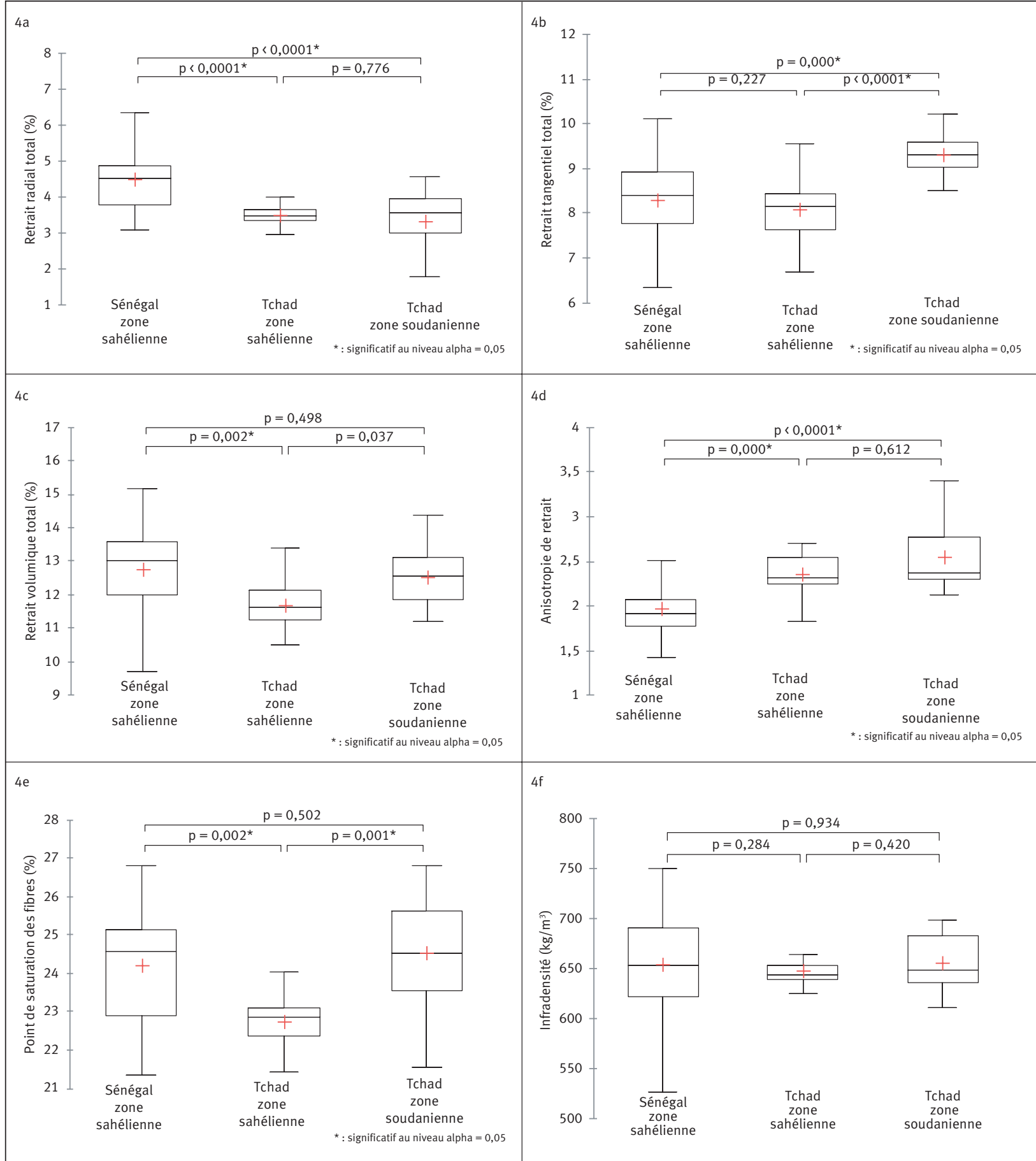

Figure 4.

Distributions des propriétés du bois de Balanites aegyptiaca en fonction de la provenance. 4.a. Comparaison du retrait radial total pour les trois provenances. $4 \mathrm{~b}$. Comparaison du retrait tangentiel total pour les trois provenances. $4 \mathrm{c}$. Comparaison du retrait volumique total pour les trois provenances. $4 \mathrm{~d}$. Comparaison de l'anisotropie de retrait pour les trois provenances. $4 \mathrm{e}$. Comparaison du point de saturation des fibres pour les trois provenances. $4 \mathrm{f}$. Comparaison de l'infradensité pour les trois provenances. Distributions of wood properties of Balanites aegyptiaca as a function of provenance. 4.a. Comparison of total radial shrinkage for the three provenances. $4 \mathrm{~b}$. Comparison of total tangential shrinkage for the three provenances. $4 \mathrm{c}$. Comparison of total volumetric shrinkage for the three provenances. $4 \mathrm{~d}$. Comparison of shrinkage anisotropy for the three provenances. $4 \mathrm{e}$. Comparison of fibre saturation point for the three provenances. 4f. Comparison of infradensity for the three provenances. 


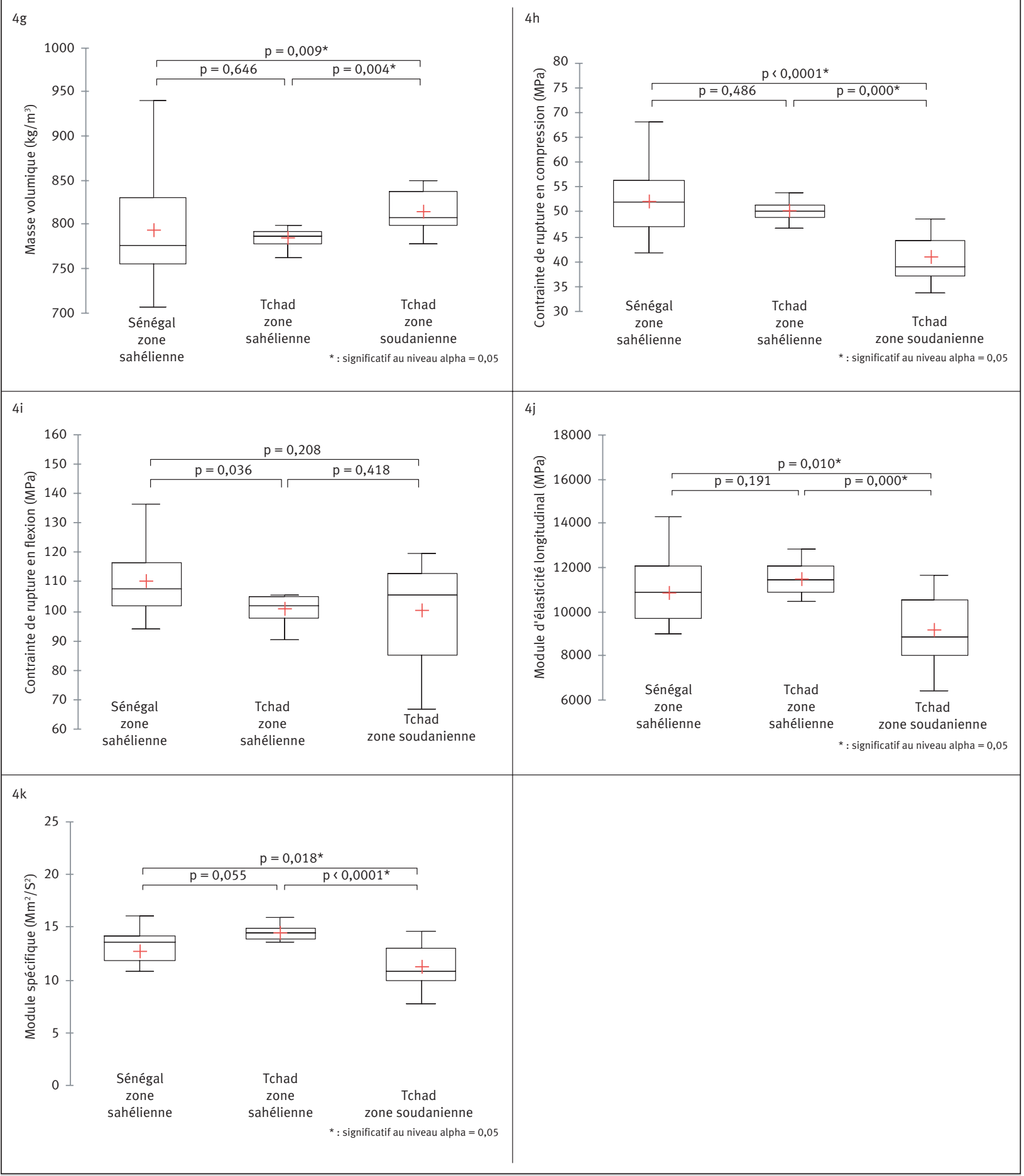

Figure 4 (suite).

Distributions des propriétés du bois de Balanites aegyptiaca en fonction de la provenance. 4g. Comparaison de la masse volumique pour les trois provenances. $4 \mathrm{~h}$. Comparaison de la contrainte de rupture en compression pour les trois provenances. $4 \mathrm{i}$. Comparaison de la contrainte de rupture en flexion pour les trois provenances. 4j. Comparaison du module d'élasticité longitudinal pour les trois provenances. 4k. Comparaison du module spécifique pour les trois provenances.

Distributions of wood properties of Balanites aegyptiaca as a function of provenance. $4 \mathrm{~g}$. Comparison of density for the three origins. 4h. Comparison of compression strength at break for the three provenances. 4i. Comparison of bending strength for the three provenances. 4j. Comparison of longitudinal modulus of elasticity for the three provenances. $4 \mathrm{k}$. Comparison of specific modulus of elasticity for the three provenances. 
Quelle que soit la provenance, les modules spécifiques moyens obtenus apparaissent très faibles pour les arbres échantillonnés : $11,5 \mathrm{Mm}^{2} / \mathrm{s}^{2}$ en zone tchadienne soudanienne, $14,7 \mathrm{Mm}^{2} / \mathrm{s}^{2}$ en zone tchadienne sahélienne et 13,6 $\mathrm{Mm}^{2} / \mathrm{s}^{2}$ en zone sahélienne sénégalaise. Ces faibles valeurs sont bien indicatrices d'arbres de savane (arbres courts et peu élancés). Pour comparaison, sur 3110 séries d'essais physiques et mécaniques de la base de données Bois du Cirad correspondant à la caractérisation de plus de 1000 espèces, seules 191 sont associées à un module spécifique inférieur à $15 \mathrm{Mm}^{2} / \mathrm{s}^{2}$, soit $6 \%$ des essais. Le module spécifique moyen dans cette base de données est de $21,5 \mathrm{Mm}^{2} / \mathrm{s}^{2}$.

\section{Qualification des bois de B. aegyptiaca étudiés par rapport à d'autres provenances}

En se référant à la grille de qualification des bois proposée dans l'Atlas des bois tropicaux (Gérard et al., 2016), le bois de $B$. aegyptiaca peut être classé comme un bois mi-lourd à lourd (masse volumique moyenne voisine de $800 \mathrm{~kg} / \mathrm{m}^{3}$ ) avec une stabilité dimensionnelle moyenne : l'anisotropie de retrait est supérieure à $2(2,2)$, le retrait radial total et le retrait tangentiel total sont moyens, respectivement égaux à $4 \%$ et 8,5\%.

Le bois de $B$. aegyptiaca présente des contraintes de rupture en compression et en flexion moyennes, respectivement de 49,4 MPa et 104,5 MPa, et un module d'élasticité longitudinal faible (10 $473 \mathrm{MPa}$ ). Le module spécifique moyen est très faible $\left(13,1 \mathrm{Mm}^{2} / \mathrm{s}^{2}\right)$, ce qui est caractéristique d'arbres de savane.

Comme mentionné en introduction, les caractéristiques physiques et mécaniques du bois de $B$. aegyptiaca ont été très peu étudiées, l'essentiel des travaux sur les utilisations de cette espèce ayant porté sur les produits forestiers non ligneux. Trois sources de comparaison à nos résultats ont été identifiées (tableau III) : Mohammed (2019), Weber et Montes (2010), et Langbour et al. (2019). Pour les deux der- nières études, les seules données disponibles concernent la masse volumique.

Pour les bois du Soudan (Mohammed, 2019), les résultats obtenus sont du même ordre de grandeur que ceux des bois prélevés au Tchad et au Sénégal pour la masse volumique $\left(780 \mathrm{~kg} / \mathrm{m}^{3}\right.$ contre $799 \mathrm{~kg} / \mathrm{m}^{3}$ et $793 \mathrm{~kg} / \mathrm{m}^{3}$ respectivement pour les bois du Tchad et du Sénégal), la contrainte de rupture en compression (47,5 MPa contre respectivement $45,5 \mathrm{MPa}$ et $52 \mathrm{MPa}$ ) et la contrainte de rupture en flexion statique $(107,1 \mathrm{MPa}$ contre respectivement 100,4 et $110,1 \mathrm{MPa}$ ). Ces mesures ont été effectuées à des taux d'humidité différents : bois stabilisés à $20^{\circ} \mathrm{C}$ et $65 \%$ d'humidité relative pour les provenances Tchad et Sénégal, et annoncés stabilisés à $7 \%$ pour la provenance Soudan. Cependant, l'impact de cette différence de taux d'humidité de stabilisation sur les résultats reste négligeable.

En revanche, on observe pour la provenance Soudan un module d'élasticité longitudinal plus faible (8 $813 \mathrm{MPa}$ contre respectivement $10337 \mathrm{MPa}$ et $10897 \mathrm{MPa}$ ) et des retraits de séchage plus élevés (5,6 \% contre 3,4\% et 4,5\% pour le retrait radial, $11,5 \%$ contre $8,7 \%$ et $8,3 \%$ pour le retrait tangentiel).

Par rapport aux bois étudiés au Tchad et au Sénégal, les bois du Soudan apparaissent donc moins stables et présentent une rigidité plus faible.

Pour onze provenances nigériennes, Weber et Montes (2010) ont obtenu une masse volumique moyenne de $627,2 \mathrm{~kg} / \mathrm{m}^{3}$, inférieure aux résultats obtenus sur les bois du Tchad, du Sénégal et du Soudan. Pour cette même caractéristique, les résultats de Langbour et al. (2019) sont de $630 \mathrm{~kg} / \mathrm{m}^{3}$ et $860 \mathrm{~kg} / \mathrm{m}^{3}$ pour deux provenances du Sénégal, $780 \mathrm{~kg} / \mathrm{m}^{3}$ et $820 \mathrm{~kg} / \mathrm{m}^{3}$ pour deux provenances du Niger, $840 \mathrm{~kg} / \mathrm{m}^{3}$ pour une provenance du Maroc et $760 \mathrm{~kg} /$ $\mathrm{m}^{3}$ pour une provenance du Burkina Faso. Chaque résultat correspond à une mesure sur une seule plaquette de bois. La masse volumique du bois de $B$. aegyptiaca apparaît donc éminemment variable en fonction de sa provenance.

\section{Tableau III.}

Comparaison des résultats obtenus sur les provenances Tchad et Sénégal par rapport aux provenances Soudan et Niger.

Comparison of the results obtained on the Chad and Senegal provenances versus the Sudan and Niger provenances.

Retrait radial total (\%)

Retrait tangentiel total (\%)

Masse volumique $\left(\mathrm{kg} / \mathrm{m}^{3}\right)$

Module d’élasticité longitudinal (MPa)

Module spécifique $\left(\mathrm{Mm}^{2} / \mathrm{s}^{2}\right)$

Contrainte de rupture en compression parallèle (MPa)

Contrainte de rupture en flexion statique (MPa)

\begin{tabular}{|c|c|c|c|}
\hline Tchad & Sénégal & Soudan* & Niger** \\
\hline 3,4 & 4,5 & 5,6 & \\
\hline 8,7 & 8,3 & 11,5 & \\
\hline 799 & 793 & 780 & 627,2 \\
\hline 10337 & 10897 & 8813 & \\
\hline 13,1 & 13,6 & 11,30 & \\
\hline 45,5 & 52 & 47,5 & \\
\hline 100,4 & 110,1 & 107,1 & \\
\hline
\end{tabular}

* : Mohammed (2019) ; ** : Weber et Montes (2010). 


\section{Conclusion}

Cette étude à caractère exploratoire a porté sur la détermination des principaux indicateurs de stabilité physique et de comportement mécanique du bois de Balanites aegyptiaca, et sur les variations de ces indicateurs suivant les zones de prélèvement : deux zones climatiques tchadiennes, la zone sahélienne et la zone soudanienne, et la zone sahélienne sénégalaise.

Les bois de $B$. aegyptiaca sont commercialisés et utilisés localement pour des usages multiples à vocations domestique, agricole et culturelle. Du fait de sa distribution disséminée dans son aire naturelle de répartition et de son caractère clairsemé, cette espèce ne fait l'objet d'aucune exploitation industrielle.

Cependant, l'élargissement des domaines d'utilisation de son bois est envisageable, toujours à l'échelle locale, d'où la nécessité d'améliorer les connaissances sur son comportement technologique. En outre, cette espèce apparaît prometteuse dans un contexte de changement climatique.

Une comparaison des résultats obtenus en fonction des zones de prélèvement a mis en évidence des tendances variables suivant les caractéristiques étudiées, que ce soit pour les indicateurs de stabilité physique (masse volumique et infradensité, retraits radial, tangentiel et volumique totaux, et point de saturation des fibres) ou ceux portant sur le comportement mécanique des bois (module d'élasticité longitudinal en flexion, contrainte de rupture en flexion statique et contrainte de rupture en compression longitudinale). Néanmoins, les caractéristiques déterminées sur les bois des trois provenances montrent que cette espèce peut être utilisée de façon appropriée sous forme de matériau pour une large gamme d'emplois.

En termes de perspectives, les différences liées à la provenance des bois selon les propriétés étudiées pourraient être expliquées en réalisant des mesures et essais complémentaires portant sur des différences de structure anatomique et de composition chimique des bois, et en ciblant des arbres pour lesquels l'âge peut être identifié.

Ainsi, la comparaison des angles des microfibrilles des bois suivant leur provenance, caractéristique liée aux conditions et à la vitesse de croissance des arbres, pourrait permettre d'expliquer les variations observées pour la masse volumique, d'une part, et les caractéristiques mécaniques, d'autre part. Le module spécifique est un bon indicateur de l'angle des microfibrilles mais l'analyse de ses variations n'a pas permis d'expliquer les différences observées entre les provenances. Les mesures d'angle des microfibrilles restent donc une caractéristique à étudier.

De même, la détermination comparée des taux d'extractibles et de leur composition selon la provenance des bois pourrait permettre de mieux comprendre l'origine des variations de retrait de séchage et les différences de tendance observées entre le retrait radial et le retrait tangentiel. En effet, la présence et la nature de ces extractibles influent directement sur le retrait du bois durant le séchage. De plus, la connaissance de ces caractéristiques chimiques permettrait de mieux comprendre l'origine de la bonne durabilité du bois de $B$. aepgyptiaca constatée dans son aire de production et d'utilisation.
Une meilleure compréhension de l'influence des conditions de croissance sur les propriétés du bois de $B$. aegyptiaca passe aussi par l'étude de bois d'autres régions où cette espèce est très présente : Nord-Cameroun, Égypte, Algérie, Zimbabwe, Israël, Arabie saoudite.

Cependant, le développement de l'emploi du bois de $B$. aegyptiaca ne peut être envisagé sans une gestion drastique de ses peuplements associée à un mode d'exploitation suffisamment rémunérateur pour les agriculteurs, et incitatif pour éviter que les arbres ne soient coupés trop jeunes pour la production de charbon.

En effet, malgré une stratégie de reproduction et des traits fonctionnels en faveur du maintien de cette espèce en milieu semi-aride, les peuplements naturels sont fragilisés par le phénomène de désertification et par la pression anthropique, et doivent faire l'objet d'une gestion adaptée pour assurer leur restauration. En l'état, ils ne peuvent faire l'objet que d'une exploitation très limitée à un niveau local.

\section{Références bibliographiques}

Adamou S., Abdoul-Salam A. I., Aissetou D. Y., 2020. Caractérisation de la population de Balanites aegyptiaca (L.) Del. et la perception de son potentiel socioéconomique dans la partie Sud-Ouest du Niger. International Journal of Biological and Chemical Sciences, 14 (5) : 1698-1715. https://www.ajol.info/index.php/ijbcs/article/ view/199689

Andersen G. L., Krzywinski K., 2007. Mortality, recruitment and change of desert tree populations in a hyper-arid environment. PLoS One, 2 (2): e208. https://journals.plos.org/plosone/ article?id=10.1371/journal.pone.0000208

Bernus E., 1979. L'arbre et le nomade. Journal d'Agriculture Traditionnelle et de Botanique Appliquée, $26^{\mathrm{e}}$ année, bulletin $\mathrm{n}^{\circ} 2,103$ 128. https://doi.org/10.3406/jatba.1979.3793

Bordonné P. A., 1989. Module dynamique et frottement intérieur dans le bois. Mesures sur poutres flottantes en vibrations naturelles. Thèse de doctorat en sciences du bois, Institut national polytechnique de Lorraine, France, 109 p.

Creac'h P., 1940. Le Balanites ægyptiaca. Ses multiples applications au Tchad. Journal d'Agriculture Traditionnelle et de Botanique Appliquée, 20 (228) : 578-593. https://www.persee.fr/doc/jatba_03703681_1940_num_20_228_1576

Dendoncker M., Ngom D., Vincke C., 2015. Trees dynamics (19552012) and their uses in the Senegal's Ferlo region: insights from a historical vegetation database, local knowledge and field inventories. Bois et Forêts des Tropiques, 326 (4): 25-41. https://doi. org/10.19182/bft2015.326.a31281

Ganaba S., Ouadba J. M., Bognounou O., 2004. Plantes de construction d'habitations en région sahélienne. Bois et Forêts des Tropiques, 282 (2) : 11-17. https://revues.cirad.fr/index.php/BFT/ article/view/20215

Gérard J., Guibal D., Cerre J.-C., Paradis S., et al., 2016. Atlas des bois tropicaux - Caractéristiques technologiques et utilisations. Versailles, France, Éditions Quæ, 1002 p.

Goffner D., Sinare H., Gordon L. J., 2019. The Great Green Wall for the Sahara and the Sahel Initiative as an opportunity to enhance resilience in Sahelian landscapes and livelihoods. Regional Environmental Change, 19: 1417-1428. https://doi.org/10.1007/ s10113-019-01481-z 
Gonzalez P., 2001. Desertification and a shift of forest species in the West African Sahel. Climate Research, 17 (2): 217-228. https:// www.int-res.com/abstracts/cr/v17/n2/p217-228/

Guibal D., Langbour P., Gérard J., 2015. Propriétés physiques et mécaniques des bois. In : Mille G., Louppe D. (éds). Mémento du forestier tropical. Versailles, France, Éditions Quæ, 873-884. http:// www.quae.com/fr/r4730-memento-du-forestier-tropical.html

Hall J. B., 2004. Balanites aegyptiaca. Enzyklopädie der Holzgewächse: Handbuch und Atlas der Dendrologie, 1-6. https:// onlinelibrary.wiley.com/doi/abs/10.1002/9783527678518. ehg1995017

Hall J. B., 1992. Ecology of key African multipurpose tree species, Balanites aegyptiaca (Balanitaceae): The state of knowledge. Forest Ecology and Management, 50 (1-2): 1-30. https://doi. org/10.1016/0378-1127(92)90311-V

Hounlonon M. C., Kouchade C. A., Kounouhewa B. B., 2017. Propriétés physiques et mécaniques du bois de teck de provenances tanzanienne et locale au Bénin. Bois et Forêts des Tropiques, 331 (1) : 45-53. https://doi.org/10.19182/bft2017.331.a31325

Iroha N. B., Hamilton-Amachree A., 2019. Inhibition and adsorption of oil extract of Balanites aegyptiaca seeds on the corrosion of mild steel in hydrochloric acid environment. World Scientific News, 126: 183-197. http://yadda.icm.edu.pl/yadda/element/bwmeta1.element.psjd-1003c17b-daef-4284-ba75-6447fbf36132

Khamis G., Saleh A. M., Habeeb T. H., Hozzein W. N., Wadaan M. A., Papenbrock J., et al., 2020. Provenance effect on bioactive phytochemicals and nutritional and health benefits of the desert date Balanites aegyptiaca. Journal of Food Biochemistry, 44: e13229. https://doi.org/10.1111/ifbc.13229

Khamis G., Schaarschmidt F., Papenbrock J., 2015. Effect of water deficiency on different genotypes of Balanites Aegyptiaca. Procedia Environmental Sciences, 29: 49-50. https://doi.org/10.1016/j. proenv.2015.07.150.

Kokutse A. D., Brancheriau L., Chaix G., 2010. Rapid prediction of shrinkage and fibre saturation point on teak (Tectona grandis) wood based on near-infrared spectroscopy. Annals of Forest Science, 67 (4): 403. https://www.afs-journal.org/articles/forest/ abs/2010/04/f09144/f09144.html?mb=1

Langbour P., Gérard J., Guibal D., Teissier du Cros R., 2008. Caractéristiques technologiques de Swietenia macrophylla King planté à la Martinique. Bois et Forêts des Tropiques, 298 (4) : 3-12. https:// revues.cirad.fr/index.php/BFT/article/view/20363

Langbour P., Paradis S., Thibaut B., 2019. Description of the Cirad wood collection in Montpellier, France, representing eight thousand identified species. Bois et Forêts des Tropiques, 339 (1) : 7-16. https://doi.org/10.19182/bft2019.339.a31709

Le Floc'h E., Aronson J., 2013. Les arbres des déserts : enjeux et promesses. Arles, France, Éditions Actes Sud, 372 p.

Mohammed N. E. A. A., 2019. Relationship between anatomical properties and some physical and mechanical properties for five wood species growing in North Darfur-Sudan. Doctoral dissertation, Sudan University of Science \& Technology. http://repository.sustech.edu/handle/123456789/23688

Morel H., Nicolini E., Bossu J., Blanc L., Beauchêne J., 2017. Qualité et usages du bois de cinq espèces forestières adaptées à la plantation à vocation de bois d'œuvre et testées en Guyane française. Bois et Forêts des Tropiques, 334 (4) : 61-74. https://doi.org/10.19182/ bft2017.334.a31492

Nitiema L. W., Sombié P. A., Koala M., Del Fiore A., 2020. Phytochemical composition and antioxidant activity of Balanites aegyptiaca, Securidaca longepedunculata and Acacia gourmaensis used against seed-borne fungi in Burkina Faso. Current Journal of Applied Science and Technology, 39 (1): 79-87. https://www.journalcjast. com/index.php/CJAST/article/view/30483
Parameswaran N., Conrad H., 1982. Wood and bark anatomy of Balanites aegyptiaca in relation to ecology and taxonomy. IAWA Journal, 3 (2): 75-88. https://doi.org/10.1163/22941932-90000755

Segla K. N., Kokutse A. D., Adjonou K., Langbour P., Chaix G., Guibal D., et al., 2015. Caractéristiques biophysiques du bois de Pterocarpus erinaceus (Poir.) en zones guinéenne et soudanienne au Togo. Bois et Forêts des Tropiques, 324 (2) : 51-64. https://doi. org/10.19182/bft2015.324.a31266

Singh Y., Sharma A., Singh N. K., Chen W. H., 2020. Development of bio-based lubricant from modified desert date oil (Balanites aegyptiaca) with copper nanoparticles addition and their tribological analysis. Fuel, 259: 116259. https://doi.org/10.1016/j. fuel.2019.116259

Thibaut B., 2016. Tree biomechanics and the transition from juvenile to mature wood. IAWS Annual Conference, Wood Science for the Future, Paris, France, 1-3 June 2016, 20 p. https://hal.archives-ouvertes.fr/hal-01784796

Weber J. C., Montes C. S., 2010. Correlations and clines in tree growth and wood density of Balanites aegyptiaca (L.) Delile provenances in Niger. New Forests, 39 (1): 39-49. https://link.springer. com/article/10.1007/s11056-009-9153-8

\begin{tabular}{|c|c|}
\hline \multicolumn{2}{|c|}{ Dougabka et al. - Contribution des auteurs } \\
\hline Rôle du contributeur & Noms des auteurs \\
\hline Conceptualisation & D. Dougabka, J. Gérard \\
\hline Gestion des données & D. Dougabka, J. Gérard \\
\hline Analyse formelle & J. Gérard, D. Dougabka, R. Marchal \\
\hline $\begin{array}{l}\text { Acquisition } \\
\text { du financement }\end{array}$ & J. Gérard, T. Bianzeube, D. Dougabka \\
\hline $\begin{array}{l}\text { Enquête et } \\
\text { investigation }\end{array}$ & $\begin{array}{l}\text { D. Dougabka, T. Bianzeube, } \\
\text { M. Dendoncker, C. Vincke, D. Guibal, } \\
\text { A. Guyot }\end{array}$ \\
\hline Méthodologie & D. Dougabka, J. Gérard, R. Marchal \\
\hline Gestion de projet & J. Gérard \\
\hline Ressources & $\begin{array}{l}\text { J. Gérard, D. Dougabka, T. Bianzeube, } \\
\text { M. Dendoncker, C. Vincke, D. Guibal, } \\
\text { A. Guyot, R. Marchal }\end{array}$ \\
\hline Supervision & J. Gérard, R. Marchal, D. Guibal \\
\hline Validation & $\begin{array}{l}\text { D. Dougabka, J. Gérard, T. Bianzeube, } \\
\text { M. Dendoncker, C. Vincke }\end{array}$ \\
\hline Visualisation & D. Dougabka, J. Gérard \\
\hline $\begin{array}{l}\text { Écriture - Préparation } \\
\text { de l'ébauche originale }\end{array}$ & $\begin{array}{l}\text { D. Dougabka, J. Gérard, T. Bianzeube, } \\
\text { M. Dendoncker, C. Vincke, D. Guibal }\end{array}$ \\
\hline $\begin{array}{l}\text { Écriture - Révision } \\
\text { et édition }\end{array}$ & D. Dougabka, J. Gérard \\
\hline
\end{tabular}

Bois et Forêts des Tropiques - Revue scientifique du Cirad . (c) Bois et Forêts des Tropiques (c) Cirad
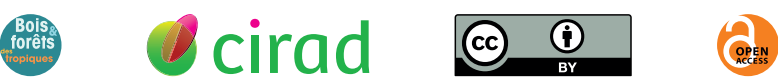

Cirad - Campus international de Baillarguet, 34398 Montpellier Cedex 5, France - Contact : bft@cirad.fr - ISSN : L-0006-579X 